Research Report Statistical Research Unit

Göteborg University

Sweden

\title{
Testing for multivariate heteroscedasticity
}

\section{Thomas Holgersson \\ Ghazi Shukur}

Research Report 2003:1

ISSN 0349-8034

Mailing address:

Statistical Research Unit

P.O. Box 660

SE 40530 Göteborg

Sweden
Fax Phone

Nat: 031-773 $1274 \quad$ Nat: 031-773 1000

Int: +46317731274 Int: +46317731000
Home Page:

http://www.stat.gu.se/stat 


\title{
Testing for Multivariate Heteroscedasticity
}

By

H.E.T. Holgersson

Department of Statistics

Göteborg university

SE-405 30 Göteborg

Sweden
Ghazi Shukur

Jönköping International

Business school

SE-55 111 Jönköping

Sweden

\begin{abstract}
In this paper we propose a testing technique for multivariate heteroscedasticity, which is expressed as a test of linear restrictions in a multivariate regression model. Four test statistics with known asymptotical null distributions are suggested, namely the Wald $(W)$, Lagrange Multiplier $(L M)$, Likelihood Ratio $(L R)$ and the multivariate Rao $F$-test. The critical values for the statistics are determined by their asymptotic null distributions, but also bootstrapped critical values are used. The size, power and robustness of the tests are examined in a Monte Carlo experiment. Our main findings are that all the tests limit their nominal sizes asymptotically, but some of them have superior small sample properties. These are the $F, L M$ and bootstrapped versions of $W$ and $L R$ tests.
\end{abstract}

Keywords: heteroscedasticity, hypothesis test, bootstrap, multivariate analysis. 


\section{Introduction}

In the last few decades a variety of methods has been proposed for testing for heteroscedasticity among the error terms in e.g. linear regression models. The assumption of homoscedasticity means that the disturbance variance should be constant (or homoscedastic) at each observation. Tests against heteroscedasticity are frequently used in many branches of applied statistics, such as quality control, biometrics and econometrics, and there exists a fair amount of heteroscedasticity tests of which all have their own pros and cons. The commonly applied White (1980) test uses a regression of squared residuals on all products and cross-products of the explanatory variables. This is not feasible in studies with small or moderate sample sizes, especially when the number of explanatory variables is large and causes considerable reduction in the degrees of freedom. For similar reasons, one cannot use the Goldfeld and Quandt (1965) test for heteroscedasticity since it is based on dividing the sample into two (possibly more) different groups, one corresponding to large values of the data and the other corresponding to small values. Another large sample test is that known as Bartlett's test, which involves dividing the error terms into $k$ groups and estimating the error variances in each group. This test and the Goldfeld-Quandt test require a natural division of the data to be made, i.e. different regimes or different groups.

On the other hand, the White test and other tests, such as the Ramsey's RESET test Ramsey (1969), the Glejser (1969) test, the Breusch-Pagan (BP) test (Breusch and Pagan (1979)) all have some implicit assumption regarding the form of heteroscedasticy in the sense that the error variance could be a function of some unknown variable(s) and, hence, these tests use different proxies for that unknown function. The $B P$ test implies finding reasonable explanatory variables, an unspecified function of which models the possible heteroscedasticity. Following Bickel (1978) a plausible approach is to regress the squared residuals from the restricted model on powers of the predictions from the same model. This test is a very general test in that it covers a wide range of heteroscedastic situations.

The above tests are, however, only strictly applicable in a single equation environment. Many models are expressed in terms of multivariate models (sometimes 
referred to as systems of equations), due to the fact that the different marginal models are connected to each other. Treating each equation separately, and performing a succession of single equation misspecification tests, may lead to the problem of mass significance and to reduction of the validity of the conclusions. The analysis of systems of equations, and in particular of allocation models has been addressed by Bewley (1986), who among other things investigated traditional tests of parameter restrictions. In general, misspecification testing is quite uncommon in multivariate models, which may partly be due to the lack of availability of a standard methodology. A few exceptions are to be found, however. Edgerton et al. (1996) use systemwise testing extensively in their analysis of the demand for food in the Nordic countries, while Huang et al. (1993) and Shukur (1997) develop strategies for testing multiple system hypotheses. Edgerton and Shukur (1999) and Shukur and Edgerton (2002), have used Monte Carlo methods to investigate the properties of tests for autocorrelation and omitted variables respectively. Doornik (1996) examined certain properties of a test for multivariate heteroscedasticity suggested by Kelejian (1982). This test relies on the assumption that the variance is a function of a known, observable variable. This assumption is in most situations an unavailable luxury. In reality, one usually has to guess a proxy variable to the unknown variable that explains the variance.

The purpose of this study is to present a heteroscedasticity test that is more general than in the previous cases. Since heteroscedasticity testing is a vast area of statistical methodology, we previously confined ourselves to a brief description of the test methods, and further details are found in cited references. We will, however, discuss more thoroughly the problems associated with the systemwise testing for heteroscedasticity, since this topic is often only briefly mentioned, if at all, in standard textbooks. In this paper, based on the $B P$ test combined with Bickel's (1978) approach, we propose a systemwise test for heteroscedasticity. We use Monte Carlo methods to analyse the size and power of various generalisations of our test in systems ranging from one to five equations, under conditions where the error terms are both normally or non-normally distributed.

The paper is arranged as follows. In Section II we present the model we analyse, and give a formal definition of some heteroscedasticity test. In Section III we discuss 
possible choices of the functional form of heteroscedasticity. In Section IV we show how our null hypothesis may be expressed as a test of a general linear hypothesis. We show in Section V how this hypothesis may be tested, while Section VI presents the design of our Monte Carlo experiment. In Sections VII-VIII we describe the results concerning the size and power of the various tests. The conclusions of the paper are presented in the final section, IX.

\section{Model and hypothesis specification}

The model of main concern in this paper is the following linear model

$$
\mathbf{Y}=\mathbf{X} \boldsymbol{\beta}+\boldsymbol{\varepsilon}
$$

where $\mathbf{Y}$ is a $n \times P$ matrix of observations on $P$ components, $\mathbf{X}$ is a fixed $n \times k$ observation matrix, $\boldsymbol{\beta}$ is a $k \times P$ matrix of parameters and $\varepsilon$ is a $n \times P$ matrix of unobservable disturbance terms. In particular, the first column of $\mathbf{X}$ is a unit vector.

Sometimes we write $(2.1)$ as

$$
\operatorname{Vec}(\mathbf{Y})=(\mathbf{I} \otimes \mathbf{X}) \operatorname{Vec}(\boldsymbol{\beta})+\operatorname{Vec}(\boldsymbol{\varepsilon})
$$

where $V e c$ is the operator stacking the columns of a matrix into one elongated column vector and $\otimes$ denotes the Kronecker product. Our primary assumptions of (2.1) are

i. $\quad \operatorname{Plim}\left(\mathbf{X}^{\prime} \mathbf{X} / n\right)^{-1}=\boldsymbol{Q}$, a fixed finite matrix.

ii. $E\left[\varepsilon_{i} \varepsilon_{i-h}^{\prime}\right]=\mathbf{0}, h \neq 0$, i.e. zero autocorrelation.

iii. $\quad E\left[\varepsilon_{i j}^{6}\right]<\infty, i=1, \ldots, n . j=1, \ldots, P$. .

This paper concerns the use of diagnostic tests for making inferences of the structure of the covariance matrix $\Xi$, where 


$$
\boldsymbol{\Xi}:=E\left[\operatorname{Vec}(\boldsymbol{\varepsilon}) \operatorname{Vec}(\boldsymbol{\varepsilon})^{\prime}\right]=\left[\begin{array}{cccc}
\sigma_{11} \Sigma_{11} & \sigma_{12} \Sigma_{12} & \cdots & \sigma_{1 P} \Sigma_{1 P} \\
\sigma_{21} \Sigma_{21} & \sigma_{22} \Sigma_{22} & \cdots & \sigma_{2 P} \Sigma_{2 P} \\
\vdots & & \ddots & \\
\sigma_{P 1} \Sigma_{P 1} & \sigma_{P 2} \Sigma_{P 2} & \cdots & \sigma_{P P} \Sigma_{P P}
\end{array}\right]
$$

There are many relevant hypotheses concerning possible covariance structures. For example, it is sometimes assumed that the covariance matrix is equal over all marginal models, i.e. that $\Sigma_{11}=\cdots=\Sigma_{P P}$ (see Bilodeau and Brenner (1999) for test of this hypothesis). In this paper, however, we will restrict ourselves to a hypothesis of particular importance, namely $\mathrm{H}_{0}: \Sigma_{j j^{\prime}}=\mathbf{I}, j, j^{\prime} \in\{1,2, \ldots, P\}$ versus $\mathrm{H}_{\mathrm{A}}: \Sigma_{j j^{\prime}}=\Sigma_{j j^{\prime}, A}$, where the subscript $A$ refers to some known alternative. The null hypothesis states that all block matrixes $\Sigma_{j j^{\prime}}$ of (2.3) equals the identity matrix, i.e. they only differ by a scalar. Clearly, when the null is true, the covariance matrix of (2.2) simply reduces to $\Xi=\sigma_{(P \times P)} \otimes \mathbf{I}_{(n \times n)}$ where $\sigma=\left\{\sigma_{j j^{\prime}}\right\}_{j, j^{\prime}=1}^{P}$, and hence the estimate of $\operatorname{Vec}(\boldsymbol{\beta})$ reduces to OLS (see e.g. Srivastava and Giles (1987)). It is readily seen that, without further restrictions, this hypothesis will be rather complicated to test in large systems. Therefore, we will focus on the diagonal block matrixes, i.e. $\Sigma_{j j}$. Our reduced null hypothesis then becomes

$$
\begin{array}{lr}
\mathrm{H}_{0}: \Sigma_{j j}=\mathbf{I} & j \in\{1,2, \ldots, P\} \\
\mathrm{H}_{\mathrm{A}}: \Sigma_{j j}=\Sigma_{j j, A} & j \in\{1,2, \ldots, P\}
\end{array}
$$

with no constraints on $\Sigma_{j j^{\prime}}$, for $j \neq j^{\prime}$. It seems unlikely that, given that all $\Sigma_{j j}=\mathbf{I}$, there would be off-diagonal covariance matrixes such that $\Sigma_{j j^{\prime}} \neq \mathbf{I}$. Hence this simplification of the hypothesis is not very restrictive. If the complete hypothesis $\mathrm{H}_{0}: \Sigma_{j j^{\prime}}=\mathbf{I}$ has to be tested, this can be done by a slight modification of the reduced test. We will discuss this matter later on in Section III. 


\section{Functional form of heteroscedasticity}

Before we consider the actual test we will discuss the structures of the alternative block matrixes, $\Sigma_{j j, A}$. If our modelling procedure is to be feasible we must put some restrictions on the alternative covariance matrix. A common assumption in single equation models is that $V\left(\varepsilon_{i}\right)=h\left(\mathbf{Z}_{i}\right)$, where $h(\cdot)$ is some bounded function of $\mathbf{Z}_{i}$ for some fixed observable $\mathbf{Z}_{i}$. An important property of $h(\cdot)$ in the context of testing for parametric heteroscedasticity is that it contains a parametric restriction, which yields $V\left[\varepsilon_{i}\right]=\sigma^{2}>0$, when the null is true. An example when this does not hold is when $V\left[\varepsilon_{i}\right]=\gamma E\left[\varepsilon_{i}\right]^{2}$, since the variance then vanishes for $\gamma=0$. A typical parameterisation that avoids this problem is $h\left(\gamma, \mathbf{Z}_{i}\right)=\alpha+\gamma \mathbf{Z}_{i}$. The choice of $\mathbf{Z}$, however, is not obvious. Further, things become more complicated if we have a system of equations (i.e. if $P>1$ ). Following Kelejian (1982) we may write

$$
E\left[\boldsymbol{\varepsilon}_{j} \boldsymbol{\varepsilon}_{j^{\prime}}^{\prime}\right]=\Sigma_{j j^{\prime}}=\operatorname{diag}(\mathbf{Z} \gamma) \quad j, j^{\prime}=1, \ldots, P
$$

where $\gamma$ is a matrix of constants. If $\mathbf{Z}$ simply is a known observable matrix (e.g. if $\mathrm{Z}=\left[\begin{array}{lll}1 & 2 \ldots\end{array}\right]$ or a vector of numbers representing different regimes), then (3.1) imposes no serious problems. Furthermore, as we work in a systemwise environment, the dependent variables in (2.1) are assumed to be correlated. It may then be reasonable to believe that the variances between the models also affect each other. This leads us to consider a parameterisation where $\mathbf{Z}_{i j}=h\left(\mu_{i j}\right), j$ is the $j$ :th column of $\mathbf{Z}_{i}$ and $\mu_{i j}:=E\left(Y_{i j}\right)$. Now, in order to impose systemwise heteroscedasticity we will express the variance as follows

$$
V\left[\varepsilon_{i j}\right]=\alpha_{j}+h\left(\gamma_{1 j}, \mu_{i 1}\right)+\ldots+h\left(\gamma_{P j}, \mu_{i P}\right) \quad i=1, \ldots, n, \quad j=1, \ldots, P
$$

Clearly, we want $h\left(\gamma_{i j^{\prime}}, \mu_{i j}\right)$ to be positive. Two reasonable choices that fulfil this criterion are $h\left(\gamma_{j j^{\prime}}, \mu_{i j}\right)=\gamma_{j j^{\prime}} \mu_{i j}^{2}$ and $h\left(\gamma_{j j^{\prime}}, \mu_{i j}\right)=\gamma_{j j^{\prime}}\left|\mu_{i j}\right|$. However, note that in some 
models the dependent variables are always positive, in which case $h\left(\gamma_{j j^{\prime}}, \mu_{i j}\right)=\gamma_{i j^{\prime}} \mu_{i j}$ may be used. This setting have been used by Shukur (2002) and Edgerton et al. (1996). In this paper we will limit ourselves to the case when $h\left(\gamma_{j j^{\prime}}, \mu_{i j}\right)=\gamma_{j j^{\prime}} \mu_{i j}^{2}$, though all our proposed tests (to follow) may readily be altered to other specifications of $h\left(\gamma_{j j^{\prime}}, \mu_{i j}\right)$. Our parameterisation of the second moment of (2.2) then becomes

$$
\mathrm{H}_{0} \cup \mathrm{H}_{A}: E\left[\varepsilon_{i}^{\cdot 2}\right]=\mathbf{Z}_{i} \Gamma=\left[\begin{array}{ll}
1 & \mu_{i}^{\cdot 2}
\end{array}\right]\left[\begin{array}{l}
\alpha \\
\gamma
\end{array}\right]=\alpha+\mu_{i}^{\cdot 2} \gamma
$$

where $\quad \varepsilon_{i}^{\cdot 2}=\left(\varepsilon_{i 1}^{2} \ldots \varepsilon_{i P}^{2}\right), \quad \alpha=\left(\alpha_{1} \ldots \alpha_{P}\right), \quad \gamma=\left(\gamma_{1} \ldots \gamma_{P}\right) \quad$ for $\quad \gamma_{j}=\left(\gamma_{1 j} \ldots \gamma_{P j}\right)^{\prime}$, $\mu_{i}^{\cdot 2}=\left(\mu_{i 1}^{2} \ldots \mu_{i P}^{2}\right)$ and $\mu_{i j}=E\left[Y_{i j}\right]$. Note that, in what follows, $\cdot 2$ always refers to the elementwise squares.

This parameterisation becomes somewhat heavy in large systems, since it will contain $P+P^{2}$ parameters. A possible restriction on (3.3) is to constrain the cross equation parameters to be zero, i.e. $\gamma_{j j^{\prime}}=0, j \neq j^{\prime}$ or to the even simpler parameterisation $\gamma_{j j^{\prime}}=0, \gamma_{11}=\ldots=\gamma_{P P}$. Such restrictions may be useful, for instance, in very small samples. Note that if one is not interested in the qualitative question whether the variables are heteroscedastic or not, but rather considers the heteroscedasticity test as a pre-test for choosing between OLS and FGLS, then another parameterisation may perhaps be preferable. For example, one may adopt the Kelejian (1982) extension of (3.3) that $\mathrm{H}_{0} \cup \mathrm{H}_{A}: \Sigma_{j j^{\prime}}=\operatorname{diag}[\mathbf{Z} \gamma]$, a parameterisation where also the off-diagonal covariance matrixes $\Sigma_{j j^{\prime}}$ are regarded. Details on estimation of the parameters in this model are available in Kelejian (1982), Doornik (1996) and Godfrey and Wickens (1982) and will not be reproduced here. 


\section{Outline of hypothesis tests}

From (3.3), we have $E\left[\varepsilon_{i j}^{2}\right]=\alpha_{j}+\gamma_{1 j} \mu_{i 1}^{2}+\ldots+\gamma_{P j} \mu_{i p}^{2}$ where $\mu_{i j}=E\left[Y_{i j}\right]$. By adding $\varepsilon_{i j}^{2}$ on both sides of this expression we get $E\left[\varepsilon_{i j}^{2}\right]+\varepsilon_{i j}^{2}=\alpha_{j}+\gamma_{1 j} \mu_{i 1}^{2}+\ldots+\gamma_{P j} \mu_{i P}^{2}+\varepsilon_{i j}^{2}$, or equivalently,

$$
\varepsilon_{i j}^{2}=\alpha_{j}+\gamma_{1 j} \mu_{i 1}^{2}+\ldots+\gamma_{P j} \mu_{i P}^{2}+\varepsilon_{i j}^{2}-E\left[\varepsilon_{i j}^{2}\right]
$$

Putting $\delta_{i j}:=\varepsilon_{i j}^{2}-E\left[\varepsilon_{i j}^{2}\right]$ we may write this model in matrix form as

$$
\boldsymbol{\varepsilon}_{i}^{\cdot 2}=\mathbf{Z}_{i} \Gamma+\boldsymbol{\delta}_{i}
$$

where $\boldsymbol{\varepsilon}_{i}^{-2}=\left[\begin{array}{lll}\varepsilon_{i 1}^{2} & \ldots & \varepsilon_{i P}^{2}\end{array}\right], \quad \mathbf{Z}_{i}=\left[\begin{array}{lll}1 & Z_{i 1} \ldots Z_{i P}\end{array}\right]=\left[\begin{array}{lll}1 & \mu_{i 1}^{2} \ldots \mu_{i P}^{2}\end{array}\right]$ and $\boldsymbol{\delta}_{i}=\left[\begin{array}{ll}\delta_{i 1} \ldots \delta_{i P}\end{array}\right]$ is an additive error term with covariance matrix $V\left[\boldsymbol{\delta}_{i}\right]=\mathbf{\Omega}_{i}$. In our application $\boldsymbol{\varepsilon}$ is unobservable and so is $\mathbf{Z}$, but this will be relaxed for so far. Now, let $\Gamma=\left[\Gamma_{1} \ldots \Gamma_{P}\right]$ for $\Gamma_{j}^{\prime}=\left[\alpha_{j} \gamma_{1 j} \ldots \gamma_{P j}\right]^{\prime}$, where $\left\{\gamma_{1 j} \ldots \gamma_{P j}\right\}_{j=1}^{P}$ are our parameters of interest. Our null hypothesis expressed by (2.4) and (3.3) is thus determined by the parameters $\left\{\gamma_{1 j} \ldots \gamma_{P_{j}}\right\}_{j=1}^{P}$ in (4.1). The usual practice of performing tests separately for all $P$ models, and then intuitively combining the results, can be misleading due to mass significance and dependent test statistics. A simple solution to this problem can be assessed by applying systemwise tests. One convenient way to do this is to test the linear hypothesis $\mathrm{H}_{0}: \mathbf{R} \Gamma=\mathbf{r}$ in the regression model (4.1). Our null hypothesis of homoscedasticity may then be expressed as

$$
\begin{aligned}
& \left.\mathrm{H}_{0}: \Gamma=\left[\begin{array}{lllll}
\left(\alpha_{1}\right. & 0 \ldots 0
\end{array}\right)^{\prime} \ldots\left(\begin{array}{lll}
\alpha_{P} & 0 . .0
\end{array}\right)^{\prime}\right]_{((p+1) \times P)}, \text { or equivalently, } \\
& \mathrm{H}_{0}: \mathbf{R}_{\left(P_{\times}(P+1)\right)} \Gamma_{((P+1) \times P)}=\mathbf{0}_{(P \times P)},
\end{aligned}
$$


where $\mathbf{R}$ is a matrix of ones and zeros. In other words, our test of systemwise heteroscedasticity is a test of a linear hypothesis in a multivariate regression model. Now, let $\hat{\boldsymbol{\delta}}_{\mathrm{H}_{0}}\left(\boldsymbol{\varepsilon}^{\cdot 2}\right)$ denote the restricted OLS residuals of (4.1) (as a function of $\varepsilon^{\cdot 2}$ ) with the constraint $\mathbf{R} \Gamma=0$, and let $\hat{\boldsymbol{\delta}}_{\mathrm{H}_{0} \cup \mathrm{H}_{\mathrm{A}}}\left(\boldsymbol{\varepsilon}^{\cdot 2}\right)$ be the unrestricted residuals (i.e. with no constraint). Then define

$$
\begin{aligned}
& \hat{\boldsymbol{\Omega}}_{\mathrm{R}}:=\hat{\boldsymbol{\delta}}_{\mathrm{H}_{0}}^{\prime}\left(\boldsymbol{\varepsilon}^{\cdot 2}\right) \hat{\boldsymbol{\delta}}_{\mathrm{H}_{0}}\left(\boldsymbol{\varepsilon}^{\cdot 2}\right), \text { and } \\
& \hat{\boldsymbol{\Omega}}_{\mathrm{U}}:=\hat{\boldsymbol{\delta}}_{\mathrm{H}_{0} \cup \mathrm{H}_{\mathrm{A}}}^{\prime}\left(\boldsymbol{\varepsilon}^{\cdot 2}\right) \hat{\boldsymbol{\delta}}_{\mathrm{H}_{0} \cup \mathrm{H}_{\mathrm{A}}}\left(\boldsymbol{\varepsilon}^{\cdot 2}\right)
\end{aligned}
$$

as the unrestricted and restricted estimators for $\boldsymbol{\Omega}$. Then, following Judge et al. (1984), the Wald, Lagrange multiplier and likelihood ratio statistics for testing $\mathrm{H}_{0}: \mathbf{R} \boldsymbol{\Gamma}=\mathbf{0}$ are given by

$$
\begin{aligned}
& \hat{\theta}_{W}=n\left\{\hbar\left(\hat{\Omega}_{R} \hat{\Omega}_{U}^{-1}\right)-P\right\} \\
& \hat{\theta}_{L M}=n\left\{P-\hbar\left(\hat{\Omega}_{U} \hat{\Omega}_{R}^{-1}\right)\right\} \\
& \hat{\theta}_{L R}=n \ln \left\{\left|\hat{\Omega}_{R}\right| /\left|\hat{\Omega}_{U}\right|\right\}
\end{aligned}
$$

where $|\cdot|$ and $\hbar(\cdot)$ are the determinant and trace operators respectively. Following Judge et al. (1984) the null distributions of our statistics $\hat{\theta}_{W}, \hat{\theta}_{L M}, \hat{\theta}_{L R}$ to test $\mathrm{H}_{0}: \mathbf{R} \boldsymbol{\Gamma}=\mathbf{0}$ is then, asymptotically, $\chi_{\left(P^{2}\right)}^{2}$, where $P$ is the number of restrictions per equation imposed by $\mathrm{H}_{0}$, and equals the number of equations in our case. Another approximation is that given by Rao (1973), namely

$$
\hat{\theta}_{F}=\left(q / P^{2}\right)\left(\left\{\left|\hat{\Omega}_{R}\right| /\left|\hat{\Omega}_{U}\right|\right\}^{1 / s}-1\right)
$$


where $s=\sqrt{\frac{P^{4}-4}{2 P^{2}-5}}, h=\left(P^{2} / 2\right)-1$, and $q=(n-P) s-h$. Under the null hypothesis, $\hat{\theta}_{F}$ is asymptotically distributed as $F_{\left(P^{2}, q\right)}$.

It is well known that the asymptotic properties of statistics of the kind in (4.4-4.7) becomes less and less accurate in small samples as the number of equations grows, see for example Laitinen (1978). This effect is expected to be particularly serious in our case as the number of restrictions to be tested is $P^{2}$. One possibility to improve the small sample properties is to use resampling techniques. A particularly interesting procedure is the so-called residual bootstrap. This method has proved useful for improving the critical values in small samples, see Horowitz (1994) and Mantalos and Shukur (1998). We will discuss this technique further in the next section.

\section{Feasible tests for parametric heteroscedasticity}

Returning to our model (4.1), i.e. $\varepsilon_{i}^{-2}=\mathbf{Z}_{i} \boldsymbol{\Gamma}+\boldsymbol{\delta}_{i}$ treated in the previous section, we see immediately that the tests of (4.4-4.7) are not feasible, since both $\varepsilon_{i}$ and $Z_{i}$ are unobservable. We therefore replace the unobservable variables with observable proxy variables in the following way. Consider the $j$ :th equation of (4.1), i.e.

$$
\varepsilon_{i j}^{2}=\alpha_{j}+\gamma_{1 j} \mu_{i 1}^{2}+\ldots+\gamma_{P j} \mu_{i P}^{2}+\delta_{i j}
$$

The most obvious choice of an observable counterpart of $\varepsilon_{i j}$ is the OLS residual $\hat{\varepsilon}_{i j}:=Y_{i j}-\hat{Y}_{i j}$. It is readily seen that $\hat{\varepsilon}_{i j}=\varepsilon_{i j}-\mathbf{x}_{i}\left(\mathbf{X}^{\prime} \mathbf{X}\right)^{-1} \mathbf{X}^{\prime} \varepsilon_{j}$, and it my be shown that $\mathbf{x}_{i}\left(\mathbf{X}^{\prime} \mathbf{X}\right)^{-1} \mathbf{X}^{\prime} \boldsymbol{\varepsilon}_{j}$ vanishes at the rate $o\left(n^{-1 / 4}\right)$ and hence $\hat{\varepsilon}_{i j}^{2}=\varepsilon_{i j}^{2}-o\left(n^{-1 / 4}\right)$ (Appendix A1). Further, $E\left[Y_{i j}\right]$ may be replaced by $\hat{Y}_{i j}$ since $\hat{Y}_{i j}=E\left[Y_{i j}\right]+\mathbf{x}_{i}\left(\mathbf{X}^{\prime} \mathbf{X}\right)^{-1} \mathbf{X}^{\prime} \varepsilon_{j}$, or, $\hat{Y}_{i j}=\mu_{i j}+o\left(n^{-1 / 4}\right)$. In other words,

$$
\hat{\mathbf{Y}}_{i}^{\cdot 2} \Gamma=\boldsymbol{\mu}_{i}^{\cdot 2} \Gamma-o\left(n^{-1 / 4}\right) \Gamma=\boldsymbol{\mu}_{i}^{\cdot 2} \Gamma+o\left(n^{-1 / 4}\right) .
$$


Therefore, $\hat{Y}_{i j}^{2}$ may be regarded a proxy variable to $\mu_{i j}^{2}$, and we get from (5.1) and (5.2) the identity

$$
\hat{\boldsymbol{\varepsilon}}_{i}^{-2}=\mathbf{Z}_{i} \Gamma+\boldsymbol{\delta}_{i}+o\left(n^{-1 / 4}\right)
$$

where now $Z_{i j}=\hat{Y}_{i j}^{2}$ rather than $Z_{i j}=E\left[Y_{i j}^{2}\right]$. The regression model (5.3) is then an operational version of (4.1) with the cost of having an additional error term vanishing at $o\left(n^{-1 / 4}\right)$. In particular, under the normality of $\varepsilon$ we have $\lim _{n \rightarrow \infty} V\left[\hat{\boldsymbol{\varepsilon}}_{i}^{\cdot 2}\right]=2 \boldsymbol{\sigma}^{\cdot 2}$ (see Appendix B). Hence, if the primary regression (2.1) has covariances among the marginal models, then so does the secondary regression, i.e. (5.3). Proceeding exactly as in Section IV, though with equation (4.1) replaced by equation (5.3), we define our feasible test statistics as

$$
\begin{aligned}
& \tilde{\theta}_{W}=n\left\{\hbar\left(\tilde{\Omega}_{R} \tilde{\Omega}_{U}^{-1}\right)-P\right\} \\
& \tilde{\theta}_{L M}=n\left\{P-\hbar\left(\tilde{\Omega}_{U} \tilde{\Omega}_{R}^{-1}\right)\right\} \\
& \tilde{\theta}_{L R}=n \ln \left\{\left|\tilde{\Omega}_{R}\right| /\left|\tilde{\Omega}_{v}\right|\right\} \\
& \tilde{\theta}_{F}=(q / G)\left(\left\{\left|\tilde{\Omega}_{R}\right| /\left|\tilde{\Omega}_{U}\right|\right\}^{1 / s}-1\right)
\end{aligned}
$$

where $\tilde{\Omega}_{R}$ and $\tilde{\Omega}_{U}$ are the restricted and unrestricted residual covariance matrixes of (5.3).

Our proposed $L M$ test of (5.5) has some interesting analogies. For the one dimensional case, (5.5) reduces to $n$ times the uncentred $R^{2}$ from the regression $\hat{\varepsilon}_{i}^{2}=Z_{i} \Gamma+\delta_{i}$. This $n R^{2}$ is equivalent to $n$ times the uncentred $R^{2}$ from the regression $\left(\hat{\varepsilon}_{i}^{2} / \hat{\sigma}^{2}-1\right)=Z_{i} \Gamma+\delta_{i}$, as noted by Davidson and MacKinnon (1993), which in turn is known as the Koenker and Basset (1982) $(K B)$ test. The $K B$ test is commonly known 
to be robust against non-normality, as opposed to the $B P$ test, which is not. Hence, our $L M$ test of (5.5) may be viewed as a multivariate extension of the $K B$ test.

As mentioned previously, tests for multivariate restrictions usually have true sizes that seriously under- or overestimate the nominal sizes in small or moderate sample sizes. Hence we shall consider bootstrapped versions of our tests proposed above. Consider the regression model (5.3), and let $\hat{\Gamma}$ and $\hat{\boldsymbol{\delta}}_{i}$ be its OLS regression parameters and residuals, respectively. The residual bootstrap technique for testing heteroscedasticity is then given by the following algorithm:

\section{Bootstrap algorithm:}

(i) Calculate the $\hat{\boldsymbol{\delta}}_{(n \times P)}$ OLS residuals of (5.3).

(ii) Let $\hat{\boldsymbol{\delta}}_{i}^{*}=\left[\hat{\delta}_{i 1}^{*} \ldots \hat{\delta}_{i P}^{*}\right]$ denote a resample observation with replacement from $\hat{\boldsymbol{\delta}}$. Further, let $\overline{\hat{\boldsymbol{\delta}}}^{*}=\left[\overline{\hat{\delta}}_{1}^{*} \ldots \overline{\hat{\delta}}_{P}^{*}\right]$ where $\overline{\hat{\delta}}_{j}^{*}=\sum_{i=1}^{n} \hat{\delta}_{i j}^{*} / n$. The centred bootstrapped residuals are then defined as $\tilde{\boldsymbol{\delta}}_{i}^{*}=\left(\hat{\boldsymbol{\delta}}_{i}^{*}-\overline{\hat{\boldsymbol{\delta}}}^{*}\right)$. Next, define $\hat{\boldsymbol{\varepsilon}}_{i}^{\cdot 2^{*}}=\mathrm{Z}_{i} \hat{\boldsymbol{\Gamma}}_{0}+\tilde{\boldsymbol{\delta}}_{i}^{*}$ where $\hat{\boldsymbol{\Gamma}}_{0}$ is the OLS estimate of $\boldsymbol{\Gamma}$ under the null. Then $\left\{\hat{\boldsymbol{\varepsilon}}^{\cdot 2^{*}}, \mathbf{Z}\right\}$ is a residual bootstrap version of $\left\{\hat{\boldsymbol{\varepsilon}}^{\cdot 2}, \mathbf{Z}\right\}$.

(iii) Calculate the restricted and unrestricted residuals from each bootstrap sample $\left\{\hat{\boldsymbol{\varepsilon}}_{b}^{\cdot 2^{*}}, \mathbf{Z}_{b}\right\}$ and calculate the test statistic $\tilde{\theta}_{b}^{*}$.

(iv) Calculate the achieved significance level by $\hat{p}_{b o o t}=\frac{1+\#\left\{\tilde{\theta}_{b}^{*} \geq \tilde{\theta}_{O b s}\right\}_{b=1}^{B}}{B+1}$.

Details of residual bootstrap are given in Freedman (1981). Preliminary simulation results (omitted here) indicate that our $W$ and $L R$ tests, i.e. (5.4) and (5.6), have rather bad small sample properties as regards the size of the test. In contrast, the $F$ and the $L M$ tests show fairly good size properties. Hence, there is no reason for bootstrapping the $F$ and $L M$ tests, and we here only include bootstrapped versions of the $W$ and $L R$ in our simulation. These will be denoted as $W_{\mathrm{Boot}}$ and $L R_{\mathrm{Boot}}$, respectively. 


\section{The Monte Carlo design}

The finite sample properties of our tests treated in Section $V$ are unknown. It is therefore important to examine whether the actual behaviour of these tests is adequately approximated by asymptotic theory. In the absence of exact results, it is necessary to investigate the finite sample performance of the statistics by means of simulation experiments. When investigating the properties of a classical test procedure, three aspects are of prime importance. First, we wish to see if the actual size of the test is close to the nominal size (used to decide the critical region for the rejection of the null hypothesis). Given that the actual size is a reasonable approximation to the nominal size, we then wish to investigate the power of the test and the robustness of the test to violations of imposed assumptions (such as nonnormality). In general, when comparing different tests we will therefore prefer those whose (i) actual size lies close to the nominal size and, given that (i) holds, (ii) have greatest power and (iii) is least sensitive to violations of the assumptions, with respect to maintained size and power. Other relevant criteria, such as which test has the soundest theoretical basis, or which test is the simplest to perform, cannot be judged quantitatively. Therefore, we leave this aspect to be judged by the reader.

In a Monte Carlo study we calculate the estimated size by observing how many times the null is rejected in repeated samples under conditions where the null is true. However, this estimated size is associated with a source of uncertainty due to finite number of replications. Let's say we define the true size of a test at a nominal size of $5 \%$ to be "reasonable" or not severely biased when it lies between $4 \%$ and $6 \%$. Clearly then, we need the confidence limits of our simulations to be at least as narrow as $\pi \pm 1 \%$. It may be shown that, at an actual size $\pi=0.05$, and using 10000 replications, $\hat{\pi} \pm 0.005$ gives an approximate $95 \%$ confidence interval for $\pi$. Hence, if the estimated size of any of our tests exceeds the interval $0.06-0.04$, for $\pi=0.05$, we conclude that the actual size of the test systematically exceeds the nominal size. In other words, an estimate outside the above mentioned range might be viewed as being inconsistent with the assumption that the corresponding finite sample value equals its asymptotically achieved values. The calculations reported here were performed using the SAS/IML program package. 
Factors that are Held Constant in the Monte Carlo Experiment. As our parameterisation of heteroscedasticity depends on the expectation of the response variable, i.e. $V\left(\varepsilon_{i j}\right) \propto E\left[Y_{i j}\right]^{2}$, the test will depend on the regression parameters, which therefore need to be specified explicitly. The extent of heteroscedasticity depends both on the regression parameters $\boldsymbol{\beta}$ and the parameter $\Gamma$ of (5.3). In our experiment we have used the following settings for the five, two and one-dimensional systems:

Regression parameters in $\mathbf{Y}=\mathbf{X} \boldsymbol{\beta}+\varepsilon$ :

$$
\boldsymbol{\beta}_{P=5}=\left[\begin{array}{lllll}
2 & 2 & 2 & 2 & 2 \\
1 & 2 & 3 & 1 & 2 \\
2 & 3 & 1 & 2 & 3 \\
3 & 1 & 2 & 3 & 1
\end{array}\right], \quad \boldsymbol{\beta}_{P=2}=\left[\begin{array}{ll}
2 & 2 \\
1 & 2 \\
2 & 3 \\
3 & 1
\end{array}\right], \quad \boldsymbol{\beta}_{P=1}=\left[\begin{array}{l}
2 \\
1 \\
2 \\
3
\end{array}\right] .
$$

Variance parameters in $V[\varepsilon]=\mathrm{Z} \Gamma$ :

$$
\boldsymbol{\Gamma}_{P=5}=\left[\begin{array}{ccccc}
1 & 1 & 1 & 1 & 1 \\
.1 & .05 & .03 & .02 & .01 \\
.05 & .1 & .05 & .03 & .02 \\
.03 & .05 & .1 & .05 & .03 \\
.02 & .03 & .05 & .1 & .05 \\
.01 & .02 & .03 & .05 & .1
\end{array}\right], \quad \boldsymbol{\Gamma}_{P=2}=\left[\begin{array}{cc}
1 & 1 \\
.1 & .05 \\
.05 & .1
\end{array}\right], \quad \boldsymbol{\Gamma}_{P=1}=\left[\begin{array}{l}
1 \\
.1
\end{array}\right]
$$

At this point it should be stressed that these parameters have no natural meaning in terms of power, because the power of the tests will be affected by both the value of $\boldsymbol{\beta}$ and the value of $\boldsymbol{\Gamma}$. For example, holding $\boldsymbol{\Gamma}$ fixed while increasing $\boldsymbol{\beta}$, would result in increasing power. The main point of our power simulation is to be able to distinguish between different powers among our four proposed tests for a specific group of heteroscedasticity parameters, and to establish consistency of the tests. Details of factors held constant in the experiment are presented in Table I. 
Factors that vary in the Monte Carlo experiment. Several factors are expected to affect the size and power properties of our heteroscedasticity tests. Since we are relying on asymptotic properties in our models in Section $V$, the number of observations is one such prime factor. We have therefore investigated samples typical for small, medium, large and very large sizes. This is equivalent to sample sizes of between 30 and 1000 observations. Another main interest of this paper lies in the analysis of systemwise tests, and thus the number of equations to be estimated is also of central importance. As the number of equations grows the consumption time becomes longer, and we chose a system with five equations as our largest model when considering the size and power properties of the tests. Medium and small size models are represented by two equations and one equation, respectively.

As the null distributions of our test statistics (5.4-5.7) rely on the normality assumption, it will be of great interest to examine their robustness to non-normality. Therefore, we shall consider non-normal as well as normal distributions of the disturbances. We will make use of one multivariate skew distribution, one symmetric heavy-tailed distribution and one normal distribution. The normal distributed variate is defined by

$$
\boldsymbol{\varepsilon}_{N}^{\prime}:=\mathbf{L}_{(P \times P)} \mathbf{\eta}_{(P \times n)}
$$

where $\eta_{i j}^{i \text { i...d. }} \sim N(0,1)$ and $\mathbf{L}$ is the Choleski root of a covariance matrix $\Sigma$, i.e. $\boldsymbol{\Sigma}=\mathbf{L} \mathbf{L}^{\prime}$. Our multivariate skew variable is defined as follows: Let $\operatorname{Gamma}(\lambda, \varphi)$ denote a gamma distribution with location parameter $\lambda$ and scale parameter $\varphi$. The skew distribution is then defined by

$$
\boldsymbol{\varepsilon}_{K_{1}}^{\prime}:=\mathbf{L}_{(P \times P)} \boldsymbol{\eta}_{(P \times n)}
$$

where $\eta_{i j}$ i.i.d. $\operatorname{Gamma}(1,9) / \sqrt{9}$. The kurtosis of a gamma variate is given by $\beta_{2}=3+6 / \varphi$ and the skewness is given by $2 / \sqrt{\varphi}$ (see Johnson et al. (1994)). Hence the (marginal) skewness of $\varepsilon_{K_{1}}$ is $2 / 3$ and the kurtosis is $3+2 / 3$ which is a moderate skewness and kurtosis as compared to a normal variate. Our symmetric non-normal distribution is defined by

$$
\boldsymbol{\varepsilon}_{K_{2}}^{\prime}:=\mathbf{L}_{(P \times P)} \boldsymbol{\eta}_{(P \times n)}
$$


where $\eta_{i j}=\pi_{i j} Z_{i j} U_{i j}$ and $p\left(\pi_{i j}\right)=\left\{\begin{array}{ll}0.5 & \pi_{\mathrm{ij}}=1 \\ 0.5 & \pi_{\mathrm{ij}}=-1\end{array}, Z_{i j}^{i . i d .} \sim[\operatorname{Gamma}(1, .25)]^{.5}\right.$. The kurtosis of (6.3) is given by $\beta_{2}=\frac{9 \Gamma(\alpha+4 \tau) \Gamma(\alpha)}{5[\Gamma(\alpha+2 \tau)]^{2}}$ (Johnson (1987)). For our choice of parameters we get $\beta_{2}=9$, which is three times that of a normal variate. Note that all three variates have first two moments identical, namely 0 and 1 . The $\varepsilon_{K_{1}}$ and $\varepsilon_{K_{2}}$ distributions will be used in the experiment in order to examine the robustness of the heteroscedasticity tests to non-normal disturbances. Details of factors varying in the experiment are displayed in Table II below.

Table I.

Factors that are Held Constant in the Monte Carlo Experiment.

\begin{tabular}{|l|c|}
\hline \multicolumn{1}{|c|}{ Factor } & Value \\
\hline Properties of $\mathbf{X}$ in repeated samples & Fixed \\
Structure of the error terms & White noise \\
Number of regressors & 3 \\
Distribution of fixed ${ }^{*}$ regressors & $U[0,1]$ \\
Regressionparameters $^{\prime}$ & $\boldsymbol{\beta}$ \\
Level of heteroscedasticity & $\boldsymbol{\Gamma}$ \\
Number of resamples & $B=99$ \\
\hline
\end{tabular}

Table II.

Factors that vary for Different Models- Size and Power Calculations.

\begin{tabular}{|l|c|c|}
\hline \multicolumn{1}{|c|}{ Factor } & Symbol & Value \\
\hline Number of observations & $\mathrm{N}$ & $30,40,60,100,200,500,1000$. \\
Distrubution of disturbances & $\boldsymbol{\varepsilon}$ & $\boldsymbol{\varepsilon}_{K_{1}}, \boldsymbol{\varepsilon}_{K_{2}}, \boldsymbol{\varepsilon}_{N}$. \\
Number of equations & $P$ & $1,2,5$. \\
\hline
\end{tabular}

\footnotetext{
*The reader may wonder why the regressors which are assumed fixed seem to be stochastic. The reason is that a considerable improvement in precision can be obtained by drawing separate samples at each replication (Edgerton (1996). Hence we save computer time by this approach.
} 


\section{Analysis of the size}

In this section we present the results of our Monte Carlo experiment concerning the size of the heteroscedasticity tests proposed in this study. We analyse the size of the tests in systems ranging from one to five equations. The size has been estimated by calculating the rejection frequencies in 10,000 replications. In this study, as mentioned earlier, we calculate the estimated size by observing how many times the null is rejected in repeated samples under conditions where the null is true. By varying factors like those described in the previous section, we can obtain a succession of estimated sizes under different conditions. In general, the closer an estimated size is to the nominal size, the better we consider a test to be. To show the main effects of the factors we discussed earlier on the performances of the tests we display the estimated sizes in our tables.

\section{VII. i. Size properties for normally distributed errors}

In this sub-section, in addition to our four tests for heteroscedasticity, we present bootstrap results for the $W$ and $L R$ tests since they have shown to behave badly in our preliminary investigation.

In Table III below we present the estimated size of our proposed heteroscedasticity tests in systems with one, two and five equations when the errors are normally distributed. The main findings are that all six tests behave well for the case of one equation system except for the $W$ test which has shown to overestimate the size in small samples. This result seems to carry over to the two-equations case with even worse results for the $W$ test and bad performance for the $L R$ in small and medium size samples. In large systems when the number of equations is equal to five, the $W$ and $L R$ tests perform badly in the sense that they over estimate the nominal size in small, medium and rather large samples, while the $L M$ test tends to underestimate it when the sample size is equal to 30 observation. The $F$ test is shown to have the best performance in almost all situations. The results also show that, when using the bootstrap technique, the $W_{\text {Boot }}$ and $L R_{\text {Boot }}$ tend to perform satisfactorily and rejecting as they should around $5 \%$ in all situations. 


\section{VII. ii. Size properties for symmetric non-normal errors}

In Table IV we present results for the size properties for symmetric non-normal errors. The table can show the robustness of our tests to a symmetric but heavy-tailed error distribution. In general, the results in the one equation case are rather similar to those when the error were normally distributed with the exception that the $L R$ is now overrejecting in small samples, i.e. the properties of the tests seem to be only slightly affected by the non-normality. However, in systems with two equations the $W$ and $L R$ tests are shown to overestimate the nominal size in small and medium size samples. This effect becomes even more accentuated in the case of five equations for almost all the tests, even the $W_{\text {Boot }}$ and $L R_{\text {Boot }}$ tend to over reject but only in small samples. Note that all tests seem to limit their nominal sizes asymptotically. The last result stands in stark contrast to the frequently used Breusch and Pagan (1979) test, which is well known to be sensitive to non-normal kurtosis in the sense that the type-one error limits $100 \%$ as the sample size increases.

Table III. Size for 1,2 and 5 equations respectively for normal distributed Errors.

\begin{tabular}{|c|c|c|c|c|c|c|}
\hline $\mathrm{N}$ & LM & LR & W & $\mathrm{F}$ & $W_{\text {Boot }}$ & $\mathrm{LR}_{\text {Boot }}$ \\
\hline 30 & 0.052 & 0.061 & 0069 & 0.050 & 0.052 & 0.050 \\
\hline 40 & 0.051 & 0.057 & ( & 0.049 & 0.051 & 0.050 \\
\hline 60 & 0.050 & 0.053 & 0.057 & 0.048 & 0.050 & 0.051 \\
\hline 100 & 0.050 & 0.052 & 0.054 & 0.049 & 0.049 & 0.049 \\
\hline 200 & 0.051 & 0.052 & 0.053 & 0.050 & 0.050 & 0.050 \\
\hline 500 & 0.050 & 0.050 & 0.050 & 0.049 & 0.050 & 0.050 \\
\hline 1000 & 0.049 & 0.049 & 0.050 & 0.049 & 0.049 & 0.051 \\
\hline $\mathrm{N}$ & LM & LR & $\mathrm{W}$ & $\mathrm{F}$ & $\mathrm{W}_{\text {Boot }}$ & $\mathrm{LR}_{\text {Boot }}$ \\
\hline 30 & 0.044 & and & 0.113 & 0.047 & 0.050 & 0.050 \\
\hline 40 & 0.045 & 0069 & r & 0.047 & 0.051 & 0.050 \\
\hline 60 & 0.047 & 00616 & $(2) 077$ & 0.048 & 0.050 & 0.050 \\
\hline 100 & 0.047 & 0.056 & 00656 & 0.047 & 0.049 & 0.050 \\
\hline 200 & 0.049 & 0.053 & 0058 & 0.049 & 0.050 & 0.050 \\
\hline 500 & 0.051 & 0.052 & 0.054 & 0.050 & 0.050 & 0.050 \\
\hline 1000 & 0.050 & 0.051 & 0.051 & 0.049 & 0.049 & 0.050 \\
\hline $\mathrm{N}$ & LM & LR & $\mathrm{W}$ & $\mathrm{F}$ & $W_{\text {Boot }}$ & $\mathrm{LR}_{\text {Boot }}$ \\
\hline 30 & 0095 & 0,249 & 0.52 / & 0.058 & 0.047 & 0.048 \\
\hline 40 & 0.041 & 0418 & 0.374 - & 0.058 & 0.050 & 0.049 \\
\hline 60 & 0.045 & 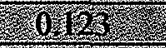 & a 25 & 0.055 & 0.050 & 0.050 \\
\hline 100 & 0.048 & $0.00 \%$ & (0) & 0.054 & 0.050 & 0.050 \\
\hline 200 & 0.050 & 0067 & VTV 0089 & 0.053 & 0.050 & 0.050 \\
\hline 500 & 0.051 & 0.057 & 0.065 . & 0.052 & 0.050 & 0.050 \\
\hline 1000 & 0.051 & 0.054 & 0.057 & 0.051 & 0.050 & 0.051 \\
\hline
\end{tabular}

The shading indicates bad performance as defined earlier, i.e. when the results lie outside the $\pm 1 \%$ interval for actual size. 
Table IV. Size for 1,2 and 5 equations respectively for $\mathrm{K}_{2}$ distributed errors.

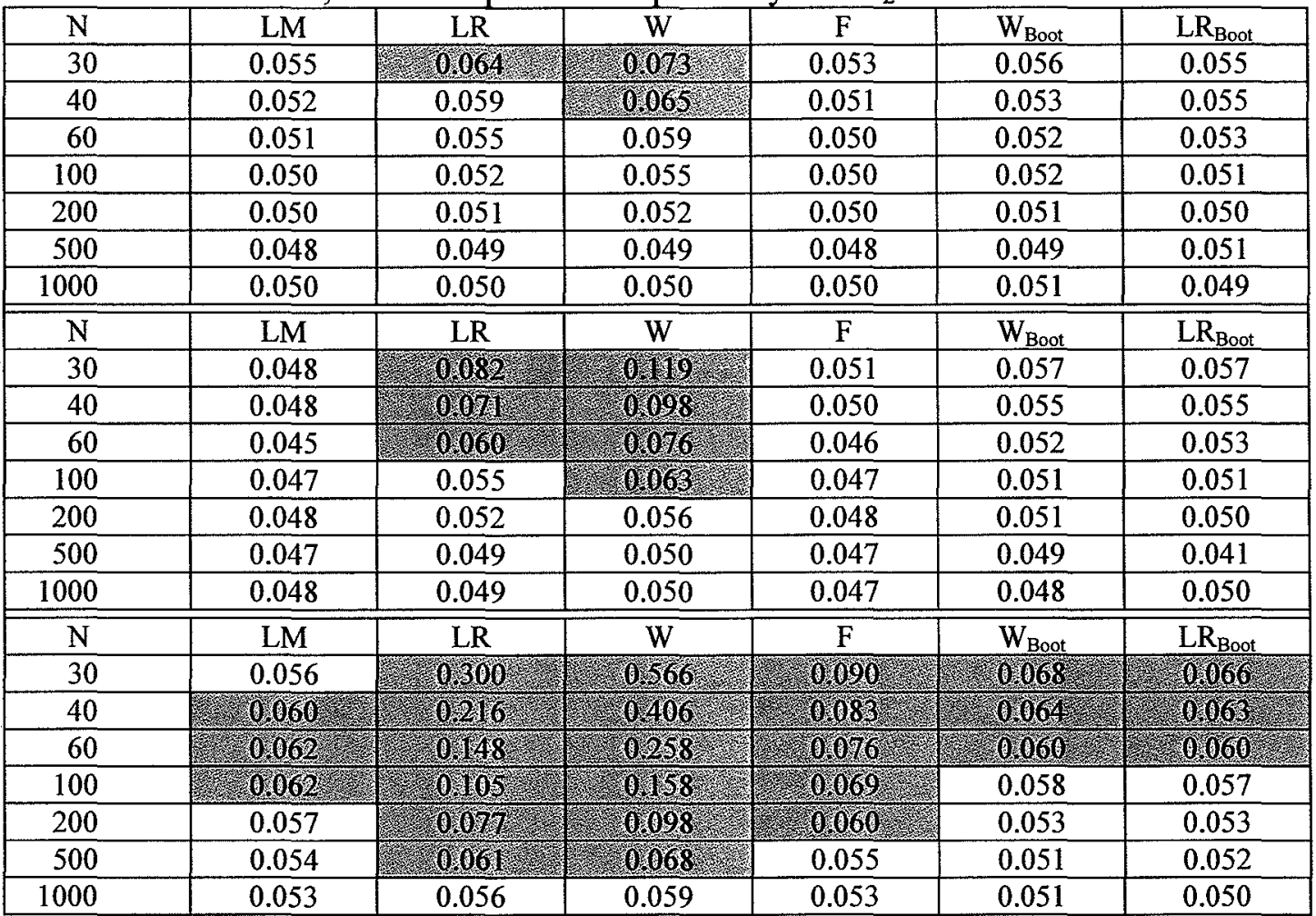

Table V. Size for 1,2 and 5 equations respectively for $\mathrm{K}_{1}$ distributed errors.

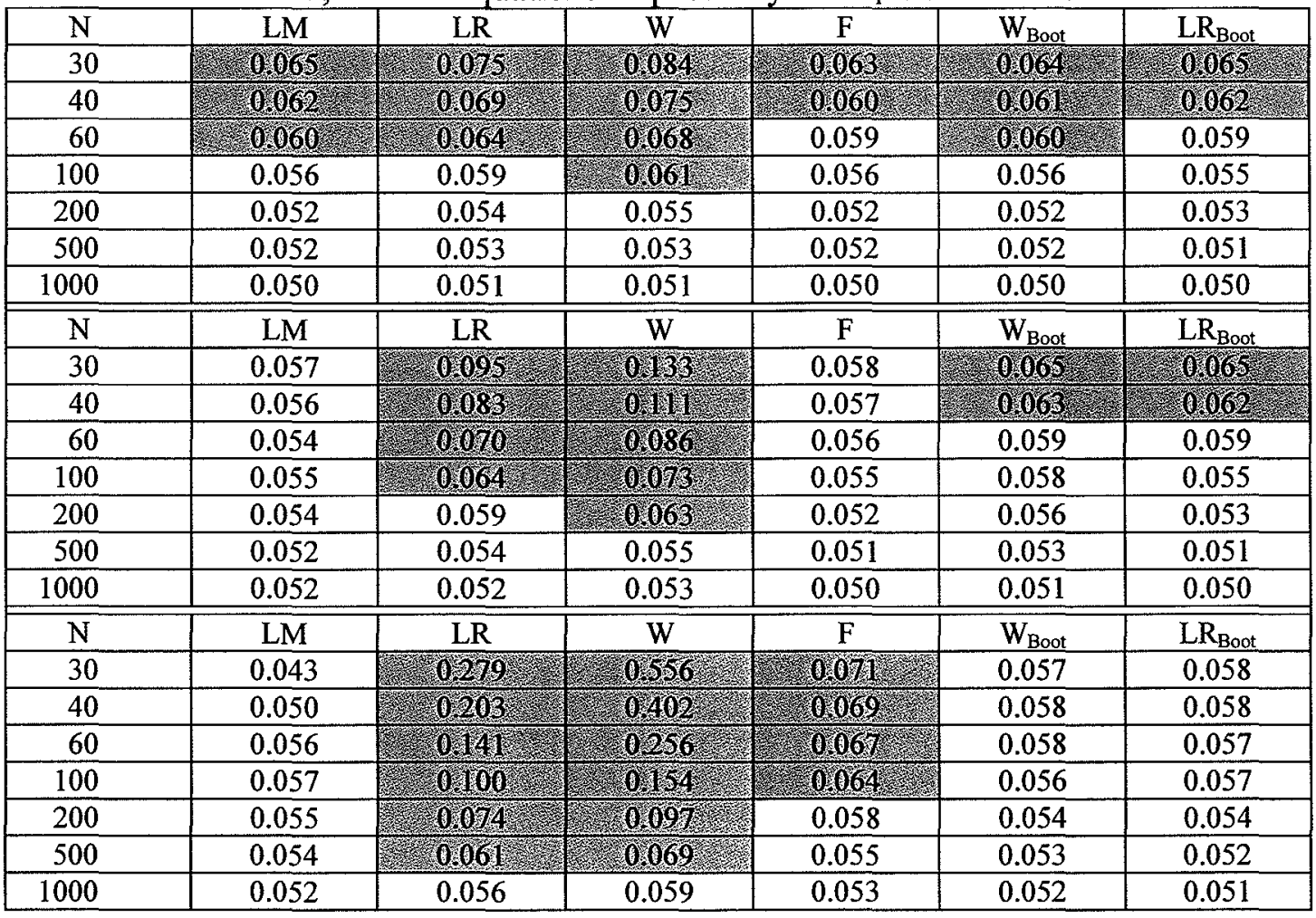

The shading indicates bad performance as defined earlier, i.e. when the results lie outside the $\pm 1 \%$ interval for actual size. 


\section{VII. iii. Size properties for skew non-normal errors}

In Table $V$ we present our results regarding the robustness to the skewed $K_{1}$ distribution. The main finding is that the $K_{1}$ distribution causes an over-rejection among the tests. All tests behave fairly well in the one equation case, but with some over rejection in small samples. Moving to the cases of two equations we see that the $L R$ and $W$ tests overestimate the size in small and medium size samples, and that the $W_{\text {Boot }}$ and $L R_{\text {Boot }}$ also over reject but only in small samples. Results from the case of five equations show that the $L R, W$ and $F$ tests overreject in small, medium and rather large samples, especially the $L R$ and $W$ where they reject around $30 \%$ and $50 \%$ of the time, respectively, under the null hypothesis. Note that, when looking at the results for the $L M$ test, it seems that, in small samples, this test performs better in the cases of two and five equations than in the case of one equation which is rather remarkable. The reason behind this can be stated as follows: It is noticeable that the $K_{1}$ distribution of errors causes a slight overrejection of the size, especially in small samples. The $L M$ test generally tends to under reject the size, especially in small samples and large systems of equations, even in those situations when the errors are either normally or non-normally distributed. Hence, an overrejection of a test that often tends to underreject the size will make it seem not to suffer severe bias.

When comparing these results with those on the previous page we find that the effect of the skewed distribution is noticeable in small samples and large systems, but disappear in large samples and small systems to be almost like the results from the symmetric non-normal error case. All tests converge to the nominal size when the sample size increases and the $W_{\mathrm{Boot}}$ and $L R_{\mathrm{Boot}}$ converge faster than the others. 


\section{Analysis of the power}

In this section we present the most interesting results regarding the power properties of the tests. We analyse the power of six versions of our heteroscedasticity test using sample sizes $30,40,60,100,200,500$ and 1000 observations. The power functions were estimated by calculating rejection frequencies from 10000 replications using the parameterisation of heteroscedasticity presented in Section VI. One could, of course, calculate and present the size-corrected power functions that give more correct information about the power of the tests. However, there is one drawback in using this method, namely that the reader can get a good idea about the real power but a misleading idea about the performance of the size (when corrected). For this reason, we decide to use the rejection rates at nominal significance levels and leave the reader to make inferential statements regarding the performances of both the size and the power. In Tables VI - VIII we present results for all the tests proposed and investigated in this study.

As shown in the previous section, $L M, F, W_{\mathrm{Boot}}$ and $L R_{\mathrm{Boot}}$ behave better than the others, and that the $W$ and $L R$ tests overreject the size. In this situation it is important to mention that even if a correctly given size is not sufficient to ensure the good performance of the power of the test, it is a prerequisite. We will therefore merely discuss the results for the $L M, F, W_{\mathrm{Boot}}$ and $L R_{\mathrm{Boot}}$ tests in this section. Power functions for the other tests are only meaningful in large samples and small systems.

In Tables VI - VIII we present the power results of our tests when the errors are normally, symmetric non-normally and skewed non-normally distributed. The power results satisfy the expected properties of increasing with the sample size to reach their maximum value of one. If we look at these tables, we cannot find any noticeable differences between the results for the different distributions. Note that when the errors are skewed non-normal, the power tends to be higher than in the other situations. 
Table VI. Power for 1,2 and 5 equations respectively for normal distributed errors.

\begin{tabular}{|c|c|c|c|c|c|c|}
\hline $\mathrm{N}$ & $\mathrm{LM}$ & $\mathrm{LR}$ & $\mathrm{W}$ & $\mathrm{F}$ & $\mathrm{W}_{\text {Boot }}$ & LR $_{\text {Boot }}$ \\
\hline 30 & 0.111 & 0.124 & $0.136 \times$ & 0.107 & 0.109 & 0.110 \\
\hline 40 & 0.143 & 0.154 & $0.163 \times$ & 0.140 & 0.139 & 0.138 \\
\hline 60 & 0.214 & 0.223 & 0.231 & 0.212 & 0.209 & 0.210 \\
\hline 100 & 0.380 & 0.387 & 0.393 & 0.378 & 0.370 & 0.371 \\
\hline 200 & 0.723 & 0.726 & 0.729 & 0.722 & 0.708 & 0.707 \\
\hline 500 & 0.991 & 0.991 & 0.991 & 0.991 & 0.989 & 0.988 \\
\hline 1000 & 1 & 1 & 1 & 1 & 1 & 1 \\
\hline \hline $\mathrm{N}$ & $\mathrm{LM}$ & $\mathrm{LR}$ & $\mathrm{W}$ & $\mathrm{F}$ & $\mathrm{W}_{\text {Boot }}$ & LR $_{\text {Boot }}$ \\
\hline 30 & 0.114 & $0.168 \times$ & $0.220 \times$ & 0.121 & 0.119 & 0.119 \\
\hline 40 & 0.148 & $0.191 \times$ & $0.233 \times$ & 0.152 & 0.151 & 0.151 \\
\hline 60 & 0.226 & $0.262 \times$ & $0.296 \times$ & 0.228 & 0.227 & 0.225 \\
\hline 100 & 0.397 & 0.424 & $0.448 \times$ & 0.399 & 0.394 & 0.395 \\
\hline 200 & 0.768 & 0.779 & $0.788 \times$ & 0.768 & 0.758 & 0.759 \\
\hline 500 & 0.998 & 0.998 & 0.998 & 0.997 & 0.997 & 0.997 \\
\hline 1000 & 1 & 1 & 1 & 1 & 1 & 1 \\
\hline \hline $\mathrm{N}$ & $\mathrm{LM}$ & $\mathrm{LR}$ & $\mathrm{W}$ & $\mathrm{F}$ & $\mathrm{W}_{\text {Boot }}$ & $\mathrm{LR}_{\text {Boot }}$ \\
\hline 30 & $0.086 \times$ & $0.405 \times$ & $0.677 \times$ & 0.139 & 0.114 & 0.111 \\
\hline 40 & 0.129 & $0.373 \times$ & $0.599 \times$ & 0.173 & 0.150 & 0.148 \\
\hline 60 & 0.220 & $0.407 \times$ & $0.573 \times$ & 0.260 & 0.238 & 0.228 \\
\hline 100 & 0.429 & $0.561 \times$ & $0.669 \times$ & 0.464 & 0.442 & 0.432 \\
\hline 200 & 0.854 & $0.892 \times$ & $0.920 \times$ & 0.868 & 0.856 & 0.847 \\
\hline 500 & 1 & 1 & $1 \times$ & 1 & 1 & 1 \\
\hline 1000 & 1 & 1 & 1 & 1 & 1 & 1 \\
\hline
\end{tabular}

Table VII. Power for 1,2 and 5 equations respectively for $K_{2}$ distributed errors.

\begin{tabular}{|c|c|c|c|c|c|c|}
\hline $\mathrm{N}$ & $\mathrm{LM}$ & $\mathrm{LR}$ & $\mathrm{W}$ & $\mathrm{F}$ & $\mathrm{W}_{\text {Boot }}$ & LR $_{\text {Boot }}$ \\
\hline 30 & 0.094 & $0.106 \times$ & $0.116 \times$ & 0.091 & 0.094 & 0.095 \\
\hline 40 & 0.103 & 0.112 & $0.121 \times$ & 0.101 & 0.104 & 0.105 \\
\hline 60 & 0.125 & 0.132 & 0.138 & 0.123 & 0.125 & 0.126 \\
\hline 100 & 0.174 & 0.179 & 0.184 & 0.173 & 0.172 & 0.176 \\
\hline 200 & 0.298 & 0.301 & 0.304 & 0.297 & 0.292 & 0.294 \\
\hline 500 & 0.621 & 0.623 & 0.624 & 0.621 & 0.608 & 0.609 \\
\hline 1000 & 0.896 & 0.896 & 0.896 & 0.896 & 0.886 & 0.887 \\
\hline \hline $\mathrm{N}$ & $\mathrm{LM}$ & $\mathrm{LR}$ & $\mathrm{W}$ & $\mathrm{F}$ & $\mathrm{W}_{\text {Boot }}$ & $\mathrm{LR}_{\text {Boot }}$ \\
\hline 30 & 0.090 & $0.139 \times$ & $0.188 \times$ & 0.096 & 0.103 & 0.102 \\
\hline 40 & 0.103 & $0.142 \times$ & $0.178 \times$ & 0.107 & 0.115 & 0.113 \\
\hline 60 & 0.126 & $0.154 \times$ & $0.181 \times$ & 0.128 & 0.136 & 0.137 \\
\hline 100 & 0.184 & 0.203 & $0.222 \times$ & 0.184 & 0.191 & 0.192 \\
\hline 200 & 0.337 & 0.349 & 0.362 & 0.336 & 0.337 & 0.340 \\
\hline 500 & 0.728 & 0.732 & 0.737 & 0.726 & 0.719 & 0.716 \\
\hline 1000 & 0.967 & 0.968 & 0.097 & 0.967 & 0.963 & 0.963 \\
\hline \hline $\mathrm{N}$ & $\mathrm{LM}$ & $\mathrm{LR}$ & $\mathrm{W}$ & $\mathrm{F}$ & $\mathrm{W}_{\text {Boot }}$ & $\mathrm{LR}$ \\
\hline 30 & $0.086 \times$ & $0.380 \times$ & $0.644 \times$ & $0.136 \times$ & $0.105 \times$ & $0.105 \times$ \\
\hline 40 & $0.105 \times$ & $0.314 \times$ & $0.521 \times$ & $0.142 \times$ & $0.113 \times$ & $0.113 \times$ \\
\hline 60 & $0.135 \times$ & $0.271 \times$ & $0.412 \times$ & $0.161 \times$ & $0.133 \times$ & $0.133 \times$ \\
\hline 100 & $0.192 \times$ & $0.282 \times$ & $0.374 \times$ & $0.211 \times$ & 0.183 & 0.183 \\
\hline 200 & 0.374 & $0.432 \times$ & $0.489 \times$ & $0.388 \times$ & 0.354 & 0.354 \\
\hline 500 & 0.853 & $0.869 \times$ & $0.883 \times$ & 0.858 & 0.838 & 0.838 \\
\hline 1000 & 0.998 & 0.998 & 0.999 & 0.998 & 0.998 & 0.998 \\
\hline
\end{tabular}

Cells marked with by an $\times$ sign indicates that the true size is too bad for the power to be meaningful. 
Table VIII. Power for 1,2 and 5 equations respectively for $K_{1}$ distributed errors.

\begin{tabular}{|c|c|c|c|c|c|c|}
\hline $\mathrm{N}$ & $\mathrm{LM}$ & $\mathrm{LR}$ & $\mathrm{W}$ & $\mathrm{F}$ & $\mathrm{W}_{\text {Boot }}$ & LR $_{\text {Boot }}$ \\
\hline 30 & $0.184 \times$ & $0.201 \times$ & $0.217 \times$ & $0.179 \times$ & $0.179 \times$ & $0.178 \times$ \\
\hline 40 & $0.225 \times$ & $0.239 \times$ & $0.253 \times$ & $0.221 \times$ & $0.219 \times$ & $0.219 \times$ \\
\hline 60 & $0.305 \times$ & $0.316 \times$ & $0.326 \times$ & 0.301 & $0.297 \times$ & 0.295 \\
\hline 100 & 0.447 & 0.455 & $0.462 \times$ & 0.445 & 0.438 & 0.440 \\
\hline 200 & 0.725 & 0.728 & 0.731 & 0.724 & 0.711 & 0.710 \\
\hline 500 & 0.979 & 0.979 & 0.980 & 0.979 & 0.975 & 0.975 \\
\hline 1000 & 1 & 1 & 1 & 1 & 1 & 1 \\
\hline \hline $\mathrm{N}$ & $\mathrm{LM}$ & $\mathrm{LR}$ & $\mathrm{W}$ & $\mathrm{F}$ & $\mathrm{W}_{\text {Boot }}$ & LR $_{\text {Boot }}$ \\
\hline 30 & 0.149 & $0.214 \times$ & $0.275 \times$ & 0.156 & $0.160 \times$ & $0.163 \times$ \\
\hline 40 & 0.190 & $0.242 \times$ & $0.291 \times$ & 0.195 & $0.199 \times$ & $0.200 \times$ \\
\hline 60 & 0.272 & $0.313 \times$ & $0.351 \times$ & 0.276 & 0.278 & 0.278 \\
\hline 100 & 0.433 & $0.461 \times$ & $0.487 \times$ & 0.434 & 0.434 & 0.436 \\
\hline 200 & 0.752 & 0.763 & $0.773 \times$ & 0.752 & 0.745 & 0.746 \\
\hline 500 & 0.993 & 0.993 & 0.993 & 0.993 & 0.991 & 0.991 \\
\hline 1000 & 1 & 1 & 1 & 1 & 1 & 1 \\
\hline \hline $\mathrm{N}$ & $\mathrm{LM}$ & $\mathrm{LR}$ & $\mathrm{W}$ & $\mathrm{F}$ & $\mathrm{W}_{\text {Boot }}$ & $\mathrm{LR}_{\text {Boot }}$ \\
\hline 30 & 0.097 & $0.426 \times$ & $0.694 \times$ & $0.155 \times$ & 0.128 & 0.128 \\
\hline 40 & 0.144 & $0.394 \times$ & $0.614 \times$ & $0.192 \times$ & 0.165 & 0.160 \\
\hline 60 & 0.230 & $0.411 \times$ & $0.571 \times$ & $0.268 \times$ & 0.240 & 0.236 \\
\hline 100 & 0.407 & $0.532 \times$ & $0.637 \times$ & $0.438 \times$ & 0.408 & 0.401 \\
\hline 200 & 0.794 & $0.841 \times$ & $0.876 \times$ & 0.809 & 0.787 & 0.777 \\
\hline 500 & 0.999 & $1 \times$ & $1 \times$ & 1 & 0.999 & 0.999 \\
\hline 1000 & 1 & 1 & 1 & 1 & 1 & 1 \\
\hline
\end{tabular}

Cells marked with by an $\times$ sign indicates that the true size is too bad for the power to be meaningful.

\section{Conclusions}

In this paper we proposed a testing technique for multivariate heteroscedasticity expressed as a test of linear restrictions in a (multivariate) model. The test is applicable in a wide class of linear models such as multiple regressions. Existing tests for multivariate heteroscedasticity are, in our opinion, either too complicated to perform and interpret or oversimplified, in the sense that they rely on unrealistic assumptions.

The proposed test is, to some extent, easy to apply and interpret. It is also (asymptotically) robust to non normality as opposed to many other tests. We therefore believe that it can provide a useful supplement to existing heteroscedasticity tests. Some relevant properties of the test have been examined in a Monte Carlo experiment. We have studied the size and power properties when the error terms 
follow a normal distribution, a symmetric non-normal distribution and a skew nonnormal distribution. This combination may cover a wide range of such departures from normality among the errors that are likely to affect the performances of the test.

A large number of models were investigated regarding the size of the test, where the distributions of the error terms, number of equations and the number of observations have been varied. For each model we have performed 10000 replications and studied six different versions of the test. The power properties have been investigated using 10000 replications per model, where, in addition to the properties mentioned above, we imposed a specific heteroscedaticity parameterisation.

When the errors follow a normal distribution, the analysis has revealed that almost all the tests perform satisfactorily regarding the size, especially in the case of single equation tests. The $W$ and $L R$ perform badly when the number of equations increases and the number of observations decreases. The results also indicate that the effect of the non-normal distributions is noticeable in small samples and large systems, but disappears in large samples and small systems to be almost identical to the results from the symmetric non-normal error case. All tests, however, converge to the nominal size when the sample size increases with the $W_{\text {Boot }}$ and $L R_{\text {Boot }}$ converging faster than the others.

As regards the power of the tests, the power functions approach the value of unity when the sample size increases. The simulation results do not indicate that the system size has any noticeable effects on the power properties. 


\section{Appendix A.}

Proposition: $\mathbf{x}_{i}\left(\mathbf{X}^{\prime} \mathbf{X}\right)^{-1}\left(\mathbf{X}^{\prime} \boldsymbol{\varepsilon}_{j}\right)=o\left(n^{-1 / 4}\right)$

Proof: By the assumption i. (p. 4) we have $\lim _{n \rightarrow \infty}\left(\mathbf{X}^{\prime} \mathbf{X} / n\right)^{-1}=Q$ where $Q=\mathrm{O}(1)$.

Now, under the null hypothesis, $V\left[\mathbf{X}^{\prime} \boldsymbol{\varepsilon}_{j} / n\right]=\mathbf{X}^{\prime} \sigma_{j j}^{2} \mathbf{I X} / n^{2}=\left(\sigma_{j j}^{2} / n\right)\left(\mathbf{X}^{\prime} \mathbf{X} / n\right)$. Since $\sigma_{j j}^{2}<\infty$, we have $\sigma_{j j}^{2}\left(\mathbf{X}^{\prime} \mathbf{X} / n\right)^{-1}=\mathrm{O}(1)$ so $V\left[\mathbf{X}^{\prime} \boldsymbol{\varepsilon}_{j} / n\right]=o\left(n^{-1 / 2}\right) \mathrm{O}(1)=o\left(n^{-1 / 2}\right)$ so $V\left[n^{1 / 4} \mathbf{x}_{i}\left(\mathbf{X}^{\prime} \mathbf{X} / n\right)^{-1}\left(\mathbf{X}^{\prime} \boldsymbol{\varepsilon}_{j} / n\right)\right]=\mathbf{x}_{i}\left(\mathbf{X}^{\prime} \mathbf{X} / n\right)^{-1} n^{1 / 2} V\left(\mathbf{X}^{\prime} \varepsilon_{j} / n\right)\left(\mathbf{X}^{\prime} \mathbf{X} / n\right)^{-1} \mathbf{x}_{i}=o(1)$.

Hence this variance limits zero asymptotically and the proposition follows.

Corollary 1: $\hat{Y}_{i j}=\mathbf{x}_{i} \hat{\boldsymbol{\beta}}_{j}=\mathbf{x}_{i}\left(\mathbf{X}^{\prime} \mathbf{X}\right)^{-1} \mathbf{X}^{\prime} \mathbf{Y}_{j}=\mathbf{x}_{i} \boldsymbol{\beta}_{j}+\mathbf{x}_{i}\left(\mathbf{X}^{\prime} \mathbf{X}\right)^{-1} \mathbf{X}^{\prime} \boldsymbol{\varepsilon}_{j}$.

Hence $\hat{Y}_{i j}=E\left[Y_{i j}\right]+o\left(n^{-1 / 4}\right)$.

Corollary 2: $\hat{\varepsilon}_{i j}=Y_{i j}-\hat{Y}_{i j}=\mathbf{x}_{i} \boldsymbol{\beta}_{j}+\varepsilon_{i j}-\mathbf{x}_{i} \boldsymbol{\beta}_{j}-\mathbf{x}_{i}\left(\mathbf{X}^{\prime} \mathbf{X}\right)^{-1} \mathbf{X}^{\prime} \boldsymbol{\varepsilon}_{j}=\varepsilon_{i j}-o\left(n^{-1 / 4}\right)$. Hence $\hat{\varepsilon}_{i j}^{2}=\varepsilon_{i j}^{2}-2 \varepsilon_{i j} o\left(n^{-1 / 4}\right)+\left\{o\left(n^{-1 / 4}\right)\right\}^{2}=\varepsilon_{i j}^{2}-o\left(n^{-1 / 4}\right)+o\left(n^{-1 / 2}\right)=\varepsilon_{i j}^{2}-o\left(n^{-1 / 4}\right)$.

\section{Appendix B.}

The asymptotic variance of (5.3) may be readily found. Consider a two-dimensional variate $\hat{\varepsilon}_{i}=\varepsilon_{i}+o\left(n^{-1 / 4}\right)$ such that $\varepsilon_{i} \sim N\left(\mathbf{0}, \boldsymbol{\sigma}^{2}\right)$ where $\boldsymbol{\sigma}^{2}=V\left[\varepsilon_{i}\right]=\left[\begin{array}{cc}\sigma_{1}^{2} & \sigma_{1} \sigma_{2} \rho \\ \sigma_{1} \sigma_{2} \rho & \sigma_{2}^{2}\end{array}\right]$.

The variance of the squared $j$ :th marginal variate, $\hat{\varepsilon}_{i j}^{2}$ say, then becomes

$$
V\left[\hat{\varepsilon}_{i j}^{2}\right]=V\left[\varepsilon_{i j}^{2}\right]+o\left(n^{-1 / 2}\right)=E\left[\varepsilon_{i j}^{4}\right]-E\left[\varepsilon_{i j}^{2}\right]^{2}+o\left(n^{-1 / 2}\right)=2 \sigma_{i}^{4}+o\left(n^{-1 / 2}\right)
$$

the last equality follows from the normality assumption. Similarly,

$$
\begin{aligned}
& \operatorname{Cov}\left[\hat{\varepsilon}_{i j}^{2} \hat{\varepsilon}_{i l}^{2}\right]=E\left[\varepsilon_{i j}^{2} \varepsilon_{i l}^{2}\right]-E\left[\varepsilon_{i j}^{2}\right] E\left[\varepsilon_{i l}^{2}\right]+o\left(n^{-1 / 2}\right)=\left(\sigma_{1}^{2} \sigma_{2}^{2}+2 \sigma_{1}^{2} \sigma_{2}^{2} \rho^{2}\right)- \\
& \sigma_{1}^{2} \sigma_{2}^{2}+o\left(n^{-1 / 2}\right)=2 \sigma_{1}^{2} \sigma_{2}^{2} \rho^{2}+o\left(n^{-1 / 2}\right) .
\end{aligned}
$$

Hence $\quad \lim _{n \rightarrow \infty} V\left[\hat{\varepsilon}_{i}^{\cdot 2}\right]=2\left[\begin{array}{cc}\sigma_{1}^{4} & \sigma_{1}^{2} \sigma_{2}^{2} \rho^{2} \\ \sigma_{1}^{2} \sigma_{2}^{2} \rho^{2} & \sigma_{2}^{4}\end{array}\right]$. 



\section{References}

Bewley, R. (1986). Allocation models, Specification, Estimation and Applications, Ballinger Publishing Company.

Bickel, P. J. (1978). "Tests for Heteroscedasticity, Nonlinearity and Nonadditivity." Annals of Statistics 6: 266-291.

Bilodeau, M. and D. Brenner (1999). Theory of Multivariate Statistics, SpringerVerlag.

Breusch, T. S. and A. R. Pagan (1979). "A Simple test for heteroscedasticity and random coefficient variation." Econometrica 47: 1287-94.

Davidson, R. and J. G. MacKinnon (1993). Estimation and Inference in Econometrics.

Doornik, J. A. (1996). "Testing Error Autocorrelation and Heteroscedasticity." Unpublished Manuscript.

Edgerton, D., B. Assarsson, et al. (1996). The Econometrics of Demand Systems. Dordrecht, Kluwer Academic Press.

Edgerton, D. and G. Shukur (1999). "Testing Autocorrelation in a System Perspective." Econometric Reviews 18(4): 343-386.

Edgerton, D. E. (1996). "Should stochastic or non-stochastic exogenous variables be used in Monte Carlo experiments?" Economics Letters 53: 153-159.

Freedman, D. A. (1981). "Bootstrapping Regression Models." The Annals of Statistics 9(6): 1218-28.

Glejser, H. (1969). "A new test for heteroscedasticity." Journal of the American Statistical Association 64: 316-23.

Godfrey, L. G. and M. R. Wickens (1982). Tests of Misspecification using locally equivalent alternative models. In Chow and Corsi: 71-99.

Goldfeld, S. M. and R. E. Quandt (1965). "Some tests for homoscedasticity." Journal of the American Statistical Association 60: 539-47.

Horowitz, J. L. (1994). "Bootstrap-based Critical Values for the Information Matrix Test." Journal of Econometric 61: 395-411.

Huang, H., A. McGuirk, et al. (1993). "Misspecification Testing and Structural Change in the Demand for Meat." Presented at the AAA meeting in Orlando.

Johnson, M. E. (1987). Multivariate Statistical Simulation. New York, Wiley. 
Johnson, N. J., S. Kotz, et al. (1994). Continous Univariate Distributions, Vol. 1., Wiley.

Judge, G. G., W. E. Griffiths, et al. (1984). The Theory and Practice of Econometrics.

Kelejian, H. H. (1982). "An Extension of a Standard Test for Heteroscedasticity." Journal of Econometrics 20: 325-33.

Koenker, R. and G. Basset (1982). "Robust tests for Heteroscedasticity Based on Regression Quantiles." Econometrica 50(1): 43-62.

Laitinen, K. (1978). "Why is Demand Homogenity so Often Rejected?" Economic letters 1: 231-3.

Mantalos, P. and G. Shukur (1998). "Size and Power of the Error Correction Model Cointegration Test. A Bootstrap Approach." Oxford Bulletin of Economics and Statistics 60(2): 249-55.

Ramsey, J. B. (1969). "Tests for specification errors in classical linear least-squares regression analysis." Journal of the Royal Statistical Society, Series B 31: 350-71.

Rao, C. R. (1973). Linear Statistical Inference. New York, Wiley.

Shukur, G. (1997). Some Aspects of Statistical Inference in Systems of Equations, $\mathrm{PhD}$ Thesis, Dept of Statistics. Lund University, Sweden.

Shukur, G. (2002). "Dynamic Specification and Misspecification in Systems of Demand Equations." Applied economics 34(6): 709-725.

Shukur, G. and D. Edgerton (2002). "The Small Sample Properties of the RESET Test as Applied to Systems of Equations." J. Statist. Comput. Simul. 72(2): 909-924.

Srivastava, V. K. and D. A. Giles (1987). Seemingly Unrelated Regression Equations Models, Marcel Dekker.

White, H. (1980). "A heteroscedastic-consistent covariance matrix and a direct test for heteroscedasticity." Econometrica 48: 421-8. 

$\underline{\text { Research Report }}$

2002:1 Frisén, M. \&

Sonesson, $\mathrm{C}$ :

2002:2 Frisén, M.:

2002:3 Jonsson, R. \& Persson, A.:

2002:4 Persson, A.:

2002:5 Persson, A.:

2002:6 Andersson, E., Bock, D. \& Frisén, M.:

2002:7 Andersson, E., Bock, D. \& Frisén, M.:

2002:8 Andersson, E., Bock, D. \& Frisén, M.:

2002:9 Holgersson, T.:
Optimal surveillance. Based on exponentially weighted moving averages.

Statistical surveillance. Optimality and methods.

Bayes prediction of binary outcomes based on correlated discrete predictors.

Prediction of work resumption among men and women with lower back- and neck pain in a Swedish population.

Prediction of work resumption in theory and practice.

Detection of turning points in business cycles.

Some statistical aspects on methods for detection of turning points in business cycles.

Statistical surveillance of cyclical processes with application to turns in business cycles.

Testing for non-normality in multivariate regression with nonspherical disturbances. 